\title{
Die plio- und pleistozänen Schotter der Wutach-Donau am Ost-Schwarzwald
}

\author{
Von Willi Paul, Vöhrenbach
}

Mit 3 Abbildungen

$\mathrm{Z}$ us ammenfassung. Die Wutach-Terrassenschotter, die den Fluß als dessen letzte Bildung vor seinem Überlaufen zum Hochrhein in der Würm-Kaltzeit zwischen seinem Austritt aus dem Gebirge und der einstigen Überlaufstelle in talab zunehmender Höhe über seiner jungen Schlucht begleiten, stellen bildungsmäßig keine Einheit („Niederterrasse“) dar. Sie weisen eine deutliche Gliederung auf in zwei glazifluviale Schotterkörper, die durch eine Diskordanz, stellenweise durch augenscheinlich nicht kaltzeitliche feinklastische Bildungen voneinander getrennt sind und sich in ihrem Konservierungsgrad merklich voneinander unterscheiden. Es wird versucht, die Schotterkörper den beiden jüngsten pleistozänen Kaltzeiten zuzuordnen. Anschließend erfolgt eine Darstellung der älteren Wutachschotter und eine Besprechung von deren mutmaßlicher Altersstellung auf der Grundlage von in den letzten Jahren erfolgten Untersuchungen.

$\mathrm{S}$ u m mary. The gravels of the "Wutach-Terrace", final sediment of the river before its running over to the Rhine and until now supposed to represent stratigraphically the Lower Terrace as a sedimentary unit, are composed of $t \mathrm{w}$ o gravel beds separated the one from the other by a distinct discordance, here and there as-it seems - even by warm time sediments with a notable discordance. Moreover both gravel beds show a different degree of conservation. The author is inclined to date the lower one as of Rissian and the upper one as of Würmian origin attributing the separating insertion of warm time origin to Eem age. The older Wutach gravel deposits are discussed with regard to their age and to paleogeographic conclusions.

\section{Einleitung}

Die Wutach - ein stattlicher Mittelgebirgsfluß mit Ursprung am Feldberg in $1100 \mathrm{~m}$ NN und Mündung in den Hochrhein bei Waldshut in etwas über $300 \mathrm{~m}$ NN nach $90 \mathrm{~km}$ langem, zunächst konsequentem, dann subsequentem Lauf über die Ostabdachung des Gebirges (Abb. 1) - hat eine lange und wechselvolle Geschichte. Ganz besonders der

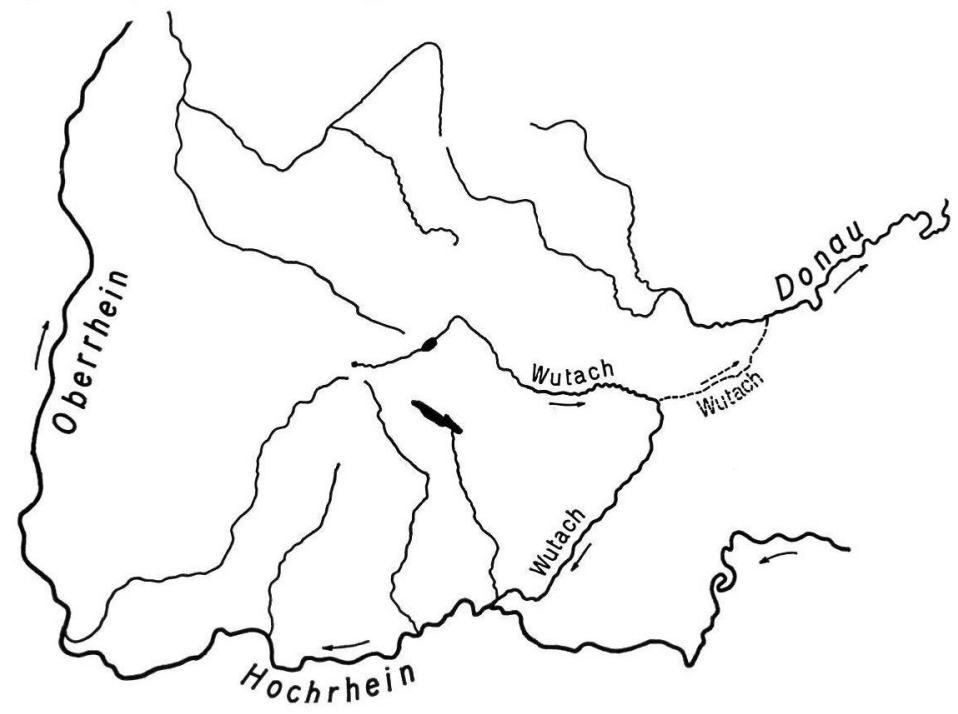

Abb. 1. Lageskizze. Gestrichelt: Verlassener danubischer Unterlauf der Wutach, aus einem Talstück der im Mittel- und Oberpliozän existierenden Aare-Donau entstanden, bis weit in die Würm-Kaltzeit hinein in Funktion, heute: Aitrachtal. Der neue Unterlauf der nunmehr rheinischen Wutach ist durch Epigenese aus einem im Oberpliozän verlassenen Laufstück der Aare-Donau entstanden. 
Umstand, daß sie in geologisch ganz junger Vergangenheit aus dem danubischen System ausgebrochen war, dem sie seit dessen Bestehen, seit dem Ausbleiben der Aare sogar als Quellfluß angehört hatte, und daß infolgedessen die ältere, danubische Tallandschaft neben der noch im Werden begriffenen jüngeren, rheinischen noch ausgezeichnet erhalten ist, hat immer wieder die Aufmerksamkeit von Geographen und Geologen zumindest auf den jüngsten Abschnitt dieser Geschichte gezogen. Die aus diesem jüngsten Abschnitt, aus der Zeit unmittelbar oder kurz vor dem Überlaufen des Flusses zum rheinischen System in ansehnlichen Mengen erhaltenen Schotter haben dabei keine auch nur etwas eingehendere Untersuchung erfahren, in der Hauptsache wohl deshalb, weil sie erst seit Beginn der fünfziger Jahre in größerem Umfange und vor allem in ihrer ganzen Mächtigkeit aufgeschlossen sind. Sie galten nicht nur morphologisch, sondern auch stratigraphisch einfach als Niederterrasse, von der man keine weiter führenden Aussagen erwartete, und so wandte sich das Interesse älteren, in nur sehr viel bescheidenerem Umfange erhaltenen Schottern zu, deren Deutung und Datierung indessen, wie auch der vorliegende Beitrag zeigt, von einem abschließenden Ergebnis noch weit entfernt sind.

\section{Die jungpleistozänen danubischen Wutachschotter}

Die soeben erwähnten und in ihrer Gesamtheit bis dahin der Würmkaltzeit zugeordneten sandigen und kiesigen Absätze unseres Flusses aus der letzten Zeit vor seinem Überlaufen zum Hochrhein begleiten ihn von seinem Austritt aus dem eigentlichen (= Grundgebirgskristallin-)Schwarzwald E von Kappel bis zu der einstigen Überlaufstelle bei Achdorf als maximal $30 \mathrm{~m}$ dicke Schotterhochböden in talab von 0 auf zuletzt $170 \mathrm{~m}$ wachsender Vertikaldistanz. Der Fluß, seit dem jüngeren Miozän ein Gefangener des Bonndorfer Grabens, verlief und verläuft hier recht genau auf und entlang der schmalen Sohlscholle dieses tektonischen, Mittleren und Südlichen Schwarzwald voneinander trennenden Gebildes. Es spricht manches, vor allem der Augenschein, dafür, daß vor oder während der Heranführung dieser selbst für danubische Morphologie, ganz besonders aber für einen Mittelgebirgsfluß abnorm mächtigen Schotter teils sockel-, teils stockwerkstektonische Sackungen den bezeichneten Abschnitt des damaligen Flußtales zum ausgesprochenen Schotterfang werden ließen, wovon heute eine größere Anzahl ausgedehnter Kiesund Sandgewinnungsbetriebe ${ }^{1}$ ) profitiert. Die von letzteren unterhaltenen, in raschem Vortrieb befindlichen Aufschlüsse lassen nun eine deutliche, in ihren wesentlichen Teilen überall, d. h. über eine Tallänge von $15 \mathrm{~km}$, unschwer wiederzuerkennende Stratigraphie erstellen:

Zuoberst erscheint eine unter Einschluß der holozänen, durchschnittlich $1 \mathrm{~m}$ messenden Verwitterungszone bis $6 \mathrm{~m}$ mächtige Lage frischen, relativ sauberen Sandkieses von der für schnellfließende Gewässer kennzeichnenden unruhigen Schichtung und auf kurze Distanz wechselnden Sortierung. Sie erscheint, entsprechend der Farbmischung frischen Grundgebirgskristallins im Einzugsgebiet (das Geröllspektrum wird von dessen Komponenten zu mehr als $95 \%$ beherrscht), aus einigem Abstand rötlichgrau getönt und ist infolge weitgehenden Mangels an bindendem Schluff in der Wand von nur geringer Standfestigkeit. Dieses Schichtglied sei hier „H a n g e n d s c h o t t e r" genannt.

Darunter folgt, i. a. die ganze restliche Mächtigkeit (maximal 10 bis $20 \mathrm{~m}$ ) der Schotterhochböden einnehmend, ein meist in sich noch weiter gegliederter Komplex von Sandkiesen, der hier als „L i e g e n d s c h o t t e r" bezeichnet wird.

In seinem unteren, durchweg mächtigeren (7 bis $15 \mathrm{~m}$ ) Teil gleicht er in Bezug auf Geröllspektrum, Schichtung und Sortierung weitgehend dem Hangendschotter in dessen jeweiliger lokaler Ausbildung (die Korngröße beider Abteilungen nimmt talab fast um

1) Das gewonnene Material kann sich i. a. gütemäßig mit jungpleistozänen Rheinkiesen nicht messen und wird vorzugsweise für Straßenbettungen verwendet; Wasserhaltung ist nicht erforderlich. 
eine Größenordnung $a b)$, ist aber minder frisch und zeigt in seinem Feinkornanteil beginnende Verwitterung, um nicht zu sagen, gelinde Verlehmung. Die Aufschlußwände sind, vermutlich infolge der hierdurch bewirkten besseren Bindung, merklich standfester, und lassen, anders als der Hangendschotter, aus einiger Entfernung ein gelb-braun-stichiges Grau als Gesamttönung erkennen.

Oben ist der Liegendschotter - bei gleichbleibendem Geröllspektrum - auf 2 bis $5 \mathrm{~m}$ Tiefe sichtlich schlechter geschichtet und sortiert, führt mitunter auch kleine, offensichtlich als Ganzes eingebettete Sandpakete, umschließt dann und wann (je weiter talauf, desto häufiger) förmliche Taschen voll feinen, in sich schräggeschichteten Feinsandes und enthält meist auch relativ und absolut großes Geröll, in bisher einem Fall (Kiesgrube Schattenmühle) auch zentnerschwere, kaum kantengerundete Granitblöcke in einer Hülle von ungeschichtetem Sand und Kies, welche über kürzeste Distanz in geordnetes Sediment überging.

Dieser obere Abschnitt des Liegendschotters ist überdies fast überall auf einen bis einige Meter Tiefe im Feinkornanteil offensichtlich infolge von oben nach unten greifender Verwitterung sehr viel stärker als das Liegende verlehmt, schluffig, ja ausgesprochen schmutzig, in der Wand unter sommerlicher Austrocknung auch bei Einschluß von ganz grobem Geröll recht standfest, und erscheint entsprechend alledem aus einiger Entferung von gelbbrauner Gesamttönung. Man hat nicht den Eindruck, daß die Untergrenze dieser Ausbildung eine eigentliche Schichtgrenze sei.

Der lebhaft fortschreitende Abbau erbrachte neuerdings in einigen Kiesgruben weiteres Detail:

In der Kiesgrube im Gewann Großwald bei Reiselfingen (8116, r 3453 460, h 5301 750) keilte genau auf der hier ohnedies durch Farbwechsel und Verlehmungsgrad besonders prägnanten Grenze zwischen Hangendschotter und Liegendschotter eine bis $2 \mathrm{~m}$ mächtige, über einige Meterzehner verfolgbare Lage eines grünlich-grauen, rostfarbig getupften oder geflammten Lehmes ein, der keine ausgesprochene Schichtung erkennen ließ.

In dem gleichen Aufschluß (8116, r 3453 700, h 53 01 800) wurde und ist noch bis jetzt eine in E-W-Richtung über $100 \mathrm{~m}$ aushaltende, im wesentlichen infolge einer Diskordanz an ihrer Obergrenze etwas an- und abschwellende, aus dem gleichen Grunde stellenweise auskeilende, ebenfalls bis $2 \mathrm{~m}$ dicke Lage eines geschichteten und sandreichen Lehmes, wiederum genau auf der Grenze Hangendschotter-Liegendschotter, angeschnitten. Thre ursprüngliche grünlichgraue Farbtönung ist vielfach, offenbar durch Oxidation, in Ocker- bis Dunkelbraun umgeschlagen.

An der gleichen Stelle drang die Abbauwand beinahe bis in das Zentrum einer steilwandigen, sichtlich einst durch Gipsauflösung in dem unterlagernden Gipskeuper entstandene Doline vor (Abb. 2). Deren Einbruch erfolgte nach Ausweis der Lagerung des Liegendschotters während der Bildung der vorhin erwähnten sandigen Lehmlage, denn diese erreicht in der Doline erheblich größere Mächtigkeit und ist von zahlreichen kleinen und kleinsten Abschiebungen durchsetzt von jener Art, wie sie für beinahe noch syngenetisch erfolgte Sackung solcher Füllungen zu erwarten ist. Hell- bis dunkelgraue, ja schwarzgraue Schichtbänderung ist ausgezeichnet erhalten. Im Kern der Füllung, stratigraphisch über dem größeren Teil der Lehmlage, steckt ein Sack verwitterten, dazu aber auch noch gebleichten Kieses, wie er ähnlich außerhalb der Doline dem höheren Abschnitt der Lehmlage eingelagert ist. Die Lehmplombe der Doline und die Lehmschicht in der Umgebung der Doline sind vielfach von heute leeren Pflanzenwurzelkanälchen durchflochten. Die Schichtung des Liegendschotters geht in Richtung auf die Doline in schmalem Bereich und raumparallel zu letzterer abwärts. Wie weit, war nicht festzustellen. Starke Regenfälle ließen das vom weiteren Abbauvortrieb umgangene Gebilde bald in sich zusammenfallen, doch sind zuvor aus allen Lagen der Lehmplombe Proben geborgen worden. Soweit sich das noch feststellen ließ, war der Hangendschotter von einem etwaigen Fortgang des Dolineneinbruchs nicht mehr betroffen worden; jedenfalls war vor dem Abräumen der Verwitterungsdecke, das dem Kiesabbau vorangeht, keine merkliche Eindellung aufgefallen. Die Hangendschotterdecke hätte demnach in der Hauptsache eine präexistente (nur noch schwache), aber nicht mehr fortgebildete Depression zu verfüllen brauchen.

In der Kiesgrube oberhalb der Schattenmühle, im Gewann Glöcklerhalde (8115, r 3448 950, h 5301 100) ist sowohl der Liegend- wie der Hangendschotter ziemlich schluffhaltig und ausgesprochen rotstichig getönt. Indessen besteht zu der hier beschriebenen Verlehmung insbesondere der Oberregion des Liegendschotters keine Beziehung. Es ist keine gelbbraune, sondern eine rot- 
braune Verfärbung, die sehr wahrscheinlich von eingeschwemmtem tonigem Detritus des Oberen Buntsandsteins aus dem Einzugsgebiet des Rötenbachs und des Reichenbächles herrührt. Sie überlagert die berichteten, stratigraphische Bedeutung besitzenden Eigentönungen und soll hier nur erwähnt werden, damit sie keine Verwirrung stiften kann. Viel wichtiger erscheint eine eigenartige



Abb. 2. Halbschematisches Profil der Wutach-Terrassenschotter im Abbau „Großwald" E Reiselfingen. $a+a^{\prime}=$ Liegendschotter, noch ca $4 \mathrm{~m}$ unter Aufschlußsohle reichend. $\mathrm{a}^{\prime}=$ durch einstige Oberflächenverwitterung veränderte Oberregion des Liegendschotters, in erster Näherung zugleich dessen Hochstandsregion. $\mathrm{b}=$ sandiger Auelehm in normaler Ausbildung und Mächtigkeit wie auch als syngenetische Plombe der Doline. $c+c^{\prime}=$ Hangendschotter. $c^{\prime}=$ dessen holozäne Verwitterungszone.

Einschaltung im unteren Teil der hier recht mächtigen Liegendschotter. Etwa $3 \mathrm{~m}$ über der damaligen Talsohle (in oberem Wellengebirge) erscheint eine halbmeterdicke, wie leicht gebleicht erscheinende Sandlage, darüber in Handhöhe ebensolcher Kies und als Abschluß eine handstarke, ockerfarbige und feinblättrige Schicht feinsandigen Lehmes. Darüber legt sich, mit scharfer Grenze, in talhangwärts und vor allem in Richtung auf ein hier einst einmündendes kleines Obermuschelkalk-Trockentälchen (noch im Aufschluß auf wenigstens $5 \mathrm{~m}$ ) anschwellender Mächtigkeit ein absolut schichtungsloser sandiger brauner Lehm, der reichlich scherbige und karrig angelöste Obermuschelkalktrümmer und daneben das vollständige Spektrum der sehr alten Wutachschotter vom Typus Göschweiler führt. Darüber setzen, mit ziemlich scharfer Grenze, wieder Sandkiese vom Typus des Liegendschotters in etwa $10 \mathrm{~m}$ Mächtigkeit ein, die wiederholt, besonders oben, grobes Geröll und kantigen Felsschutt führen, eine hangende Verlehmungszone erkennen lassen und mit deutlicher Grenze gegen die zwar etwas rotbraunen Schluff (s. o.) führenden, im übrigen aber sauberen Hangendschotter abstoßen.

Es liegt nahe, in dieser ganzen stratigraphischen Gliederung der Wutachterrassenschotter die Abbildung eines zweimaligen Wechsels von einer kalten zu einer warmen Klimaphase zu erblicken. Der Liegendschotter wäre als Vorstoßschotter bzw. - in seinem obersten Teil - als Hochstandschotter einer Kaltphase zuzuordnen, die schichtungslose Schuttund Geröllehmdecke bei der Schattenmühle als solifluidales Produkt der Frühzeit dieser gleichen Kaltphase, die ausgedehnte, eine Doline im Zuge von deren Bildung verfüllende Lehmdecke im Großwald als Auelehm und die verhältnismäßig dicke Zone beginnender Verlehmung im obersten Teil des Liegendschotters als Zersatzprodukt einer folgenden Warmphase, und schließlich der Hangendschotter als glazifluviale, in der Hauptsache wohl nur Vorstoß-Leistung einer nächsten und jüngsten Kaltphase, in deren Verlauf der Fluß zum Hochrhein überlief, von der Aufschotterung früher oder später zur Eintiefung überging, worauf mit der gegenwärtigen Warmphase kräftige, inzwischen bis zu Metertiefe vorgedrungene Verwitterung des Hangendschotters einsetzte. 
Die Größenordnung der postulierten Klimawechsel ist eine offene Frage. Der Referent neigt $\mathrm{zu}$ der Vorstellung einer zweimaligen Kaltzeit-Warmzeit-Abfolge, weil ihm die Unterschiede im Erhaltungszustand der beiden Schotterkörper - auch soweit diese in ihren tieferen Teilen keiner eigentlichen Oberflächen-Verwitterung unterworfen waren -, die Mächtigkeit jener Zersatzzone des Liegendschotters wie auch die der darüber gebreiteten Auelehmdecke ${ }^{2}$ ) für bloße Stadial- bzw. Interstadialbildungen zu groß erscheinen. Auch würde sich gegen eine Deutung als Stadial- bzw. Interstadial-Effekte die Frage erheben, ob klimatische Schwankungen dieser geringeren Größenordnung - es kämen dann ja nur solche der anscheinend doch nicht allzu kräftig gegliederten Würmkaltzeit in Betracht - in 700-800 m Meereshöhe über der Abdachung eines bis zu $1500 \mathrm{~m}$ aufragenden Mittelgebirges unserer Breiten sich in solcher Deutlichkeit abzuzeichnen vermöchten. Und schließlich würde sich dieser Frage sogleich die andere zur Seite stellen, wo dann die doch unbedingt auch für die Rißkaltzeit(en) zu erwartenden glazifluvialen Absätze zunächst einmal des hier betrachteten Flußgebietes deponiert bzw. geblieben sind. Mit der Vorstellung einer ausgedehnten Vorlandvereisung auf der E-Seite des Schwarzwaldes könnte man wenigstens für das hier betrachtete Gebiet dieser Frage ausweichen. Aber es ist bis jetzt nicht gelungen, eine solche Vorstellung auch nur durch einen einzigen Befund zu verifizieren $\left.{ }^{3}\right)$.

Zur Korrelierung der hier mitgeteilten Stratigraphie der danubischen Wutachterrassenschotter mit den Sandern und Moränen im Raum von Neustadt (Schwarzwald) besteht gegenwärtig keine Möglichkeit. Der Gutach-Wutach-Talabschnitt in der augenscheinlich noch im Jungpleistozän hier im Aufsteigen begriffen gewesenen Hochfirst-Kippscholle (PAUl 1955) bedeutet eine praktisch gänzliche Unterbrechung aller fluvialen, glazifluvialen und etwaigen glazialen Sedimentkomplexe, weil hier fast nur erodiert wurde.

Jungpleistozäne Schotter der danubischen Wutach finden sich auch auf der Sohle ihres einstigen Unterlaufes, dem heutigen Aitrachtal zwischen Blumberg und Kirchen-Hausen, bei welch letzterem Ort sie sich mit der heutigen Breg-Brigach-Donau vereinigte. Diese Schotter sind fast überall unter mächtigen Solifluktionszungen und Schwemmkegeln aus autochthonem Abtragungsschutt begraben und daher nur aus Bohrungen bekannt. Anstehend kennt man sie nur von der Talwasserscheide, von Blumberg (8117, r 3464700 , h 5300 300), wo sie ihrer leichten Gewinnbarkeit wegen immer wieder abgebaut worden sind und wo sie gegenwärtig (noch!) an der bezeichneten Stelle in einer kleinen Grube nebst Liegendem und Hangendem 6 m mächtig gut erschlossen sind. Die Schotter erscheinen hier ziemlich frisch und auch sauber und gleichen durchaus dem Hangendschotter der hier mitgeteilten Stratigraphie. Wie diesem fehlt auch ihnen jegliche Untergliederung. Wie weit dies alles für die begrabene Fortsetzung der Schotterdecke talabwärts gilt, läßt sich aus den Registern der erwähnten (Wasserversorgungs-)Bohrungen nicht feststellen; die Mächtigkeit bewegt sich nach deren Ausweis durchweg um $5 \mathrm{~m}$.

Wenn sich sonach talauf von der Überlaufstelle der Wutach hinauf bis vor die mutmaßlichen Gletscher-Maximalstände ein für mittelgebirgische Verhältnisse wohlgegliedertes Fluvialprofil präsentiert, talab hingegen nur ein recht bescheidenes (das aber der Regel auf der Außenabdachung des Schwarzwaldes entspricht), so scheint ein solcher Unterschied nicht so sehr eine Frage der Bildung der betreffenden Ablagerungen, sondern eine solche ihrer E rhalt ung zu sein. Wir dürfen doch wohl annehmen, daß über der Sohle des heute verlassenen Unterlaufes unseres Flusses zur Zeit der Bildung des Liegendschot-

2) Daß die berichtete Zersatzzone wie auch jene Lehmdecke in ihrer Mächtigkeit schwanken und letztere weithin ganz fehlt - auf die offenbare Diskordanz über beiden ist hier bereits hingewiesen worden -, dürfte im Hinblick auf die vielfältige Umlagerung in einer kaltzeitlichen Talaue, besser gesagt Schotterflur durchaus verständlich sein. 1966.

3) Pfannenstiel \& Rahm 1963a, 1963b; Paul 1965; Pfannenstiel, Rahm \& Moll 1966; Paul 
ters von weiter talauf ebenfalls aufgeschottert worden war, daß aber diese Schotter wieder so gut wie gänzlich weggeführt worden sind, noch ehe das dortige Aqquivalent des Hangendschotters abgelagert war. Die Bewahrung älterer kaltzeitlicher Schotter und diesen aufgesetzter warmzeitlicher Bildungen bis zur Überdeckung durch die jüngsten kaltzeitlichen Schotter in dem Talstück zwischen Kappel und Achdorf erscheint nicht exogen, sondern endogen bedingt zu sein (S. 233). Gleichwohl ist eine so gründliche Ausräumung der Schotter einer Kaltzeit vor Ablagerung derjenigen aus der folgenden - es handelt sich offenbar um eine Regelerscheinung bei Mittelgebirgsflüssen — bis jetzt noch nicht in völlig befriedigender Weise erklärt worden.

\section{Die vor-jungpleistozänen Wutachschotter}

Von ihnen sind, wie eingangs bemerkt, nur sehr viel bescheidenere Reste erhalten. Sie besitzen ein von dem der jungpleistozänen merklich, in ihren orographisch höher liegenden Vorkommen sogar auffallend verschiedenes Ge rölls pektrum. Diese Abwandlung des Geröllspektrums mit zunehmender Höhe über den Wutach t e r r a s s e n schottern und in diesem Fall auch mit zunehmendem Alter ist indessen nicht nur eine verwitterungsresistenzbedingte, sondern auch eine solche von paläogeographischer Bedingtheit.

Von den Komponenten karbonatischer Natur abgesehen, die talaufwärts von der Úberlaufstelle sogar im Hangendschotter überaus selten sind, werden offensichtlich die Magmatite und Metamorphite des Grundgebirgskristallins, deren Anteil in den jungpleistozänen Schottern mitunter $95 \%$ und mehr erreicht, zuerst zersetzt; am längsten vermögen anscheinend amphibolitische Gneise und quarzreiche Magmatite geeigneter Textur zu widerstehen. Nur die im NW des heutigen Wutach-Einzugsgebietes und darüber hinaus häufigeren Gänge und Knauer von weißem Quarz sind praktisch unvergänglich. Daß sie den jungpleistozänen Wutachschottern fast gänzlich fehlen, berechtigt zu der Vorstellung, daß von dorther zu dieser Zeit keine stärkere Schutteinspeisung erfolgte, weder unter kaltzeitlichen noch unter warmzeitlichen Bedingungen. Die Beteiligung (an sich als Folge anderer paläogeographischer Verhältnisse) an der Zusammensetzung der älteren Schotter ist bemerkenswert hoch (dies als Folge der langwährenden verwitterungsbedingten Auslese).

Die Komponenten aus dem Buntsandstein sind von zweierlei Art. Da sind zunächst einmal die $\mathrm{S}$ a n d ste in e als solche, von denen diejenigen mit kieseligem Bindemittel (es ist primär, also nicht erst nach Bildung und Einbettung der Gerölle zugeführt) auch in der wenig konservierungsholden Matrix dieser alten Schotter über Zeiträume von mehr als der Dauer einer känozoischen Unterabteilung hinweg erhalten bleiben könnten. Aber auch Sandsteine mit anderem, weniger beständigem Bindemittel finden sich gar nicht so selten selbst in den höchsten und ältesten Vorkommen. Das ursprüngliche Beteiligungsverhältnis aller dieser Buntsandsteine s. s. läßt sich infolge der mehr oder minder weitgehenden Zerstörung des Anteils aus Grundgebirgskristallin nicht mehr feststellen. Ohne Zweifel war es geringer als heute, aber erheblich höher als in den jungpleistozänen, in dieser Beziehung ein noch nicht durch Verwitterungsauslese verfälschtes Bild bietenden Schottern, denn im älteren Pleistozän und ganz besonders im Pliozän war der Buntsandstein auch im Südlichen und im Mittleren Schwarzwald noch weit verbreitet. Die andere dem Buntsandstein entstammende Komponente der älteren Wutachschotter sind die ob ihrer ganz ausgezeichneten Zurundung von den oben erwähnten Grundgebirgsquarzen meist eindeutig zu unterscheidenden weißen Quarz- und bunten Quarzitgerölle seiner Konglomerate. Auch sie sind praktisch unvergänglich und haben nur auf dem Weg zu ihrer zweiten Lagerstätte eine leichte weitere Abrollung erfahren, deren Ausmaß der Zurundung der nicht seltenen, noch im ursprünglichen konglomeratischen Verband durch Gebirgsklüftung zerissenen Exemplare zu entnehmen ist. Diese 





zweite Buntsandstein-Komponente fehlt den oben beschriebenen jungpleistozänen Schottern absolut - ein untrügliches negatives Merkmal selbst im aufschlußlosen Gelände. Den untersten und jüngsten vorjungpleistozänen Schottern sind sie aber bereits in nicht übersehbarem Maße beigemengt, in den höheren, älteren, großenteils nur noch als Streu erhaltenen herrschen sie oft, zumindest zahlenmäßig, vor. Quarzitgerölle von $15 \mathrm{~cm}$ Länge sind hier keine Seltenheiten. Diese Buntsandstein-Komponenten waren in der am weitesten nach SE ausholenden Grobschüttung der Gallischen Schwelle in den Schwarzwald beheimatet, von welcher noch Reste in dem gegenwärtigen distalsten Ausgehenden des Buntandsteins N und NE von dem Gebiet der oberen Wutach erhalten sind (Steinberg 7915, 8015, Stöcklewald 7915), während beispielsweise im Feldberggebiet zu der gleichen Zeit, wenn überhaupt, nur Sandstein sedimentiert wurde (cf. Alpersbacher Brekzie). Quarzitgerölle aus dem Buntsandstein auf zweiter Lagerstätte im Wutachgebiet weisen demnach mit großer Sicherheit auf Herkunft aus NW hin, wahrscheinlich sogar von außerhalb des heutigen Einzugsgebietes, wo unser Fluß vorzeiten seinen Ursprung haben mochte (BuBNOFF 1913).

Weitere resistente und ebenfalls paläogeographisch aussagefähige Komponenten sind diejenigen aus den spät- bis postdiagenetischen Silexbildungen des Mittleren Muschelkalkes: Zuckerkornquarzite, Hornsteine und Kieseloolithe. In den jungpleistozänen Wutachschottern nur vereinzelt auftretend, als Zuckerkornquarzit noch in der ursprünglichen Knauergestalt, sonst als nur wenig kantenbearbeitete Scherben, sind sie schon den nächstälteren Schottern so zahlreich beigemengt, daß man sie bereits im Acker, also ohne Aufschluß, zu Gesicht bekommt. Die Zuckerkornquarzite, volummäßig führend, innen oft noch schneeweiß, randlich gelbbraun verfärbt, können bei flüchtigem Besehen leicht für weiße oder gebleichte Gerölle aus Buntsandstein gehalten und dann in ihrer Besonderheit gar nicht wahrgenommen werden. In den höchsten und ältesten Wutachschottern ist der Anteil dieser Silexbildungen aus dem Mittleren Muschelkalk im Vergleich zu den Komponenten aus dem Buntsandstein um ein Mehrfaches größer als dem Verhältnis der Beteiligung am Aufbau des triassischen Schichtprofils entspricht. Das ist kaum anders zu deuten, als daß zur Zeit der Bildung dieser Schotter in dem damaligen Einzugsgebiet des Flusses mächtige alte Böden und Zersatzmassen aus Mittlerer Trias verhältnismäßig rasch und flächenhaft aufbereitet und weggeführt worden sind.

Trotz solcher Folgerungen aus den Geröllspektren der älteren und ganz alten Schotter im Wutachgebiet steht eine auch nur halbwegs gesicherte Datierung ihrer Bildung noch im weiten Feld. Soweit es sich überhaupt noch um dreidimensionale Vorkommen und nicht nur um im Acker abermals streng selektierte Streuen handelt, fehlt es an brauchbaren Aufschlüssen, weil Qualität und Quantität gegenwärtig keinen Abbau mehr lohnen, und die wenigen vor Jahrzehnten noch vorhandenen Aufschlüsse werden mehr und mehr ein Opfer der Müllbeseitigung oder sonstigen Fortschrittes. Ohne ein gutes Dutzend Schürfe sind auf die Dauer keine Beiträge zur Datierung und zur Deutung der Genese mehr zu erlangen.

Einzig für eine (?) nächstältere aufschotternde und wahrscheinlich klimatisch bedingte Phase der Geschichte unseres Flusses - nach der hier versuchten Zuordnung der jungpleistozänen Terrassenschotter würde es sich um Mittelpleistozän handeln - lassen sich einige allerdings schon weitgehend voneinander isolierte und vielleicht nicht nur aus einer Schotterstreu bestehende Vorkommen anführen. Allen ist das in zeitlicher Rückschau erstmalige Auftreten der bunten Quarzitgeröll-Komponenten aus den Buntsandstein-Konglomeraten, der weißen Grundgebirgengangquarze und eine stärkere Beteiligung der Silexbildungen aus dem Mittleren Muschelkalk gemeinsam neben immer noch vorherrschenden, mehr oder minder verwitterten Magmatiten und Metamorphiten. Alle liegen auch nur größenordnungsmäßig $10 \mathrm{~m}$ über der würmkaltzeitlichen Schotterflur am 
Talhangfuß der (danubischen) Wutach als bei jüngerer Ausräumung und Vertiefung des Tales verschont gebliebene Reste. Zu erwägen und in Betracht zu ziehen ist auch die Möglichkeit, daß die eben erwähnten spezifischen Komponenten dieser Schotter bereits auf dritter bzw. zweiter Lagerstätte ruhen: Sie könnten mobilisierten und abgetragenen noch älteren, sogar den ältesten hier bekannten Vorkommen entstammen.

Bis jetzt sind von dieser nächstälteren Schottergeneration die folgenden Vorkommen bekannt:

B o 11 („Boller Moräne“), Terrasse hinter der Häuserreihe E vom Dorfplatz (8116, r 3451500 , h 5299 950), 1921 von WEPFER (1924) in Rohrgräben beobachtet und als rißkaltzeitliche Moräne gedeutet, seither verschollen und 1966 wiedergefunden (PAUL 1966). Matrix aus gelbem Lehm, Mächtigkeit und Liegendes nicht bekannt, Hangendes vielleicht Fließ- und Frostschutt aus Mittlerem und Oberem Muschelkalk und dessen Detritus.

B l u mberg, am SE-Fuß des Stoberges im Aitrachtal (8117, r 3468000 , h 5301 250). Großenteils müllverfüllte ehemalige Kiesgrube. Nach ScHalch (1908) 4,5 m mächtig, Hangendes $=$ Malm-Frost- und -Fließschutt.

Le ipferdingen, am Wiedberg gegenüber dem Bahnhof (8117, r 3473 850, h 5303 650). Aufgelassene Kiesgrube, teilweise zum Sportplatz planiert, Schottermächtigkeit nach SchaLCH (1908) mindestens $4 \mathrm{~m}$, Liegendes unbekannt, Hangendes $1,5 \mathrm{~m}$ Hanglehm und Frostschutt.

A u lfingen, Gewann Niedel (8117, r 3474 700, h 5306 500), zugeackerte einstige kleine Kiesgrube, von SchaLCH (1908) erwähnt.

Die noch älteren Wutachschotter lassen sich nur ganz allgemein der großen Zeitspanne Jungpliozän + Altpleistozän zuordnen, vielleicht mit Ausnahme der ältesten und höchsten überhaupt noch erhaltenen, der bekannten Göschweiler Schotter. In den älteren und in einem Teil der jüngeren Publikationen ist ihnen „altdiluviales“ Alter als Höchstmaß zugeschrieben worden, wobei man - die lange Dauer des Altpleistozäns ist ja eine recht junge Erkenntnis - an Altere Deckenschotter, also Günzkaltzeit, dachte ${ }^{4}$ ). Einzig G. Wagner (1929) hat die Möglichkeit pliozänen Alters offengelassen. Der Referent hat diese Schotter von vornherein in das Pliozän gestellt (PAul 1949, 1958, 1965) und hält heute (PAul 1970) in Anlehnung an die Entwicklung der Aare-Donau ein oberpliozänes Alter für das wahrscheinlichste. Diese Schotter, als Lager in einer Doline auf dem Roßhag bei Göschweiler (8115, r 3449700 , h 5302 950) aufgeschlossen, zumindest als charakteristische Streu in den Gewannen Wacht, Langäcker, Lautenlinden der Markung Göschweiler, Lautenlinden, Nachtweid der Markung Seppenhofen, Lerchental, Hohegerten, Bucheck, Dauen der Markung Reiselfingen, alles auf 8115 und 8116 verbreitet, sind noch einmal besonders gekennzeichnet durch das außerordentliche Kaliber ihres Grobblockanteils (bis jetzt bekannte maximale Größenordnung: $1 \mathrm{~m} \phi$ ). Sie stehen damit und mit ihrer Lage innerhalb der Schichtstufenlandschaft der Außenabdachung des Schwarzwaldes in e in e r Reihe mit zahlreichen anderen Vorkommen, die über der NE-Seite des Gebirges als „Höhenschotter“, über seiner S- und SW-Seite als „Wanderblockformation“ beschrieben und in allernächster Nachbarschaft, von Villingen und Donaueschingen, als Laible- und Schellenberg-Schotter bekannt gemacht worden sind (PAUL 1950, 1958). Man war bis jetzt geneigt, die reiche Beteiligung gröbsten Block-Kalibers (es handelt sich dabei nur um Komponenten aus Buntsandstein) aller dieser Schotter mit effektiv (wahrscheinlich im frühen Mittel- und im frühen Oberpliozän) erfolgten ersten Aufdomungen des heutigen Schwarzwaldes in ursächlichen Zusammenhang zu bringen. Es ist indessen nicht ausgeschlossen (und der Referent hat das schon vor Jahren (PAUL 1958) geäußert), daß auch exogene Faktoren etwa in Gestalt vegetations-dezimierender Klimaverschlechterung mitgespielt haben, ja vielleicht sogar vorherrschend waren: Nach HoInKes (1968) hat die Untersuchung von Bohrkernen aus dem antarktischen Ozean „Anzeichen für eine

4) Die bei Pfannenstiel, Rahm \& Moll (1966) vertretene Datierung und Deutung der Göschweiler Schotter als rißkaltzeitliche Moräne wird dem Geröllspektrum nicht gerecht; hierzu sei auch auf BrunNaCKer (1964) verwiesen (1. c. S. 74). 
maximale Vereisung vor etwa 3 Millionen Jahren “ ergeben, auch läßt sich die oben (S. 239) aus dem Geröllspektrum der älteren und ganz besonders der Göschweiler Schotter gefolgerte geradezu exzessive Abräumung mächtiger alter Eluvialböden aus Mittlerer Trias über dem heutigen Schwarzwald mindestens ebensowohl mit exogenen wie mit endogenen Einwirkungen ursächlich in Zusammenhang bringen, eine Denkmöglichkeit, die diese ganze etwas eingehendere Betrachtung eines pliozänen Schotters in dem Jahrbuch einer vorzugsweise der Erforschung des Pleistozäns dienenden wissenschaftlichen Vereinigung rechtfertigen möge.

Im übrigen markieren die Göschweiler Schotter in ihrer oben angegebenen Verbreitung einen alten (mittelpliozänen?) Lauf der Wutach in dem damaligen Ausstrich der Grenzregion Obermuschelkalk-Kohlenkeuper, d. i. in der Niederung vor einer einstigen auf der Sohlscholle des Bonndorfer Grabens sitzenden, hier W-E verlaufenden KeuperUnterlias-Schichtstufe. Diese scheint, etwa ab Mitte Oberpliozän vielleicht, mit dem sie tragenden S Span dieser Sohlscholle in die Tiefe gegangen zu sein - mit der Abschiebung bzw. Flexur Göschweiler-Bachheim-Mundelfingen als Scharnier, wobei ihr die Wutach nach Maßgabe der gleichzeitigen endogenen und exogenen Reliefänderung (=Abwanderung der Schichtstufe) sowohl abwärts $(150 \mathrm{~m})$ als auch seitwärts (S-wärts, $2 \mathrm{~km}$ ) folgte und dann durch stärkeres, mit dem Mittelpleistozän offenbar im wesentlichen beendetes Einschneiden fixiert wurde. Diese neue Laufrichtung hat sie seither nicht mehr verlassen. Spuren dieses Ab- und Seitwärtsgleitens sind in Gestalt einiger Schotterstreuen und einer Schotterdecke noch erhalten. Sie zeigen die hier mitgeteilte Abwandlung des Geröllspektrums in der Zeit vom Mittelpliozän bis zum Mittelpleistozän recht gut, ruhen auf Obermuschelkalk als Streu, auf Kohlen- und unterstem Gips-Keuper als Decke, bedürfen aber für weitergehende Aussagen vor allem einer planvollen Erschürfung ihres einzigen lagerhaften Vorkommens, der sogenannten „Reiselfinger Moräne“ (8116, r 3451 700, h 5301 240, über den Rappenfelsen). Bei diesem ziemlich umstrittenen Vorkommen (PAul 1966) handelt es sich augenscheinlich um eine (glazi-)fluviale Anlagerung an einen N-S verlaufenden Rücken aus Obermuschelkalk mit Kohlen- und ganz wenig Gips-Keuper, der von der Wutach vorher durchschnitten worden sein muß, und um die jüngste der über dem Schotterzug Boll-Blumberg-Leipferdingen-Aulfingen (S. 240) folgenden Bildungen. Nach ihrer gegenwärtigen Verfassung5) würde man sie in Unkenntnis der Ausbildung der oben beschriebenen jungpleistozänen Terrassenschotter vielleicht als eisnahen Teil eines Sanders einer jungpleistozänen Vereisung ansprechen. Aber bei einem Vergleich mit der hier als Hochstandschotter gedeuteten Oberregion des Liegendschotter-Komplexes unter Berücksichtigung des erheblich höheren Alters und dessen Folgen (weitgehende Zersetzung nicht nur des Feinkornanteils, sondern auch der groben Fraktion des Grundgebirgeskristallins, dadurch bedingte Sackungen der ganzen Masse in sich selbst, Sackungen der Unterlage und wiederholte kaltzeitliche Kryoturbation der oberflächennahen Partien) erscheint eine Deutung als Hochstandsschotter einer älteren Vereisung als wahrscheinlicher.

Aus alledem ergibt sich ein vergleichsweise bescheidenes Ausmaß der pleistozänen Eintiefung der $\mathrm{d}$ a n u b is chen Wutach - im Durchschnitt noch keine $100 \mathrm{~m}$. Der heutige Vertikalabstand zwischen der Felsunterlage der hier in das Oberpliozän gestellten Göschweiler Schotter und des jungpleistozänen Liegend-Komplexes der Terrassenschotter über der Schattenmühle beträgt ( $900 \mathrm{~m} \mathrm{Mh}-740 \mathrm{~m} \mathrm{Mh}) 160 \mathrm{~m}$. Von diesem Betrag entfallen nach den oben (S. 241) entwickelten Vorstellungen größenordnungsmäßig $60 \mathrm{~m}$ auf sockelund stockwerkstektonische Verstellungen - junge Aufkippung der Hochfirst-Scholle zwischen den beiden Ortlichkeiten und schätzungsweise $25 \mathrm{~m}$ auf das Konto noch pliozäner Erosion. Bei Blumberg, wo die frühesten, wahrscheinlich spätpontischen Aare-Do-

5) Sie ist von Pfannenstiel \& Rahm für deren Publikation 1963 neu erschürft worden. 
nau-Schotter (auf dem Eichberg in über $900 \mathrm{NN}$ ) mehr als $200 \mathrm{~m}$ über der jungpleistozänen Talsohle (695 m NN) liegen, dürfte nach allen Gegebenheiten die Aare-Donau bei ihrem Ausbleiben auf heute rund $770 \mathrm{~m}$ Meereshöhe (Sattel von Zollhaus) eingetieft gewesen sein, woraus sich für das Mittelpliozän und für das frühe Jungpliozän eine Erosion von nahezu $150 \mathrm{~m}$ und für die Zeit von da bis in das Jungpleistozän eine solche von $75 \mathrm{~m}$ ergeben. Über Tuttlingen ist die Aare nachweisbar bei einem Stand der Erosion $100 \mathrm{~m}$ über der jungpleistozänen Felssohle ausgeblieben, so daß mit größenordnungsmäßig $75 \mathrm{~m}$ pleistozäner Eintiefung zu rechnen ist; die mittel- und früh-jungpliozäne Eintiefung ist von ungefähr der gleichen Größe wie bei Blumberg. In der Nähe der Wutach selbst können die Verhältnisse am Schellenberg bei Donaueschingen zum Vergleich herangezogen werden. Dort beträgt die Vertikaldistanz zwischen der jungpliozänen Wanderblockformation (=825 m Mh) und der jungpleistozänen Talsohle rund $150 \mathrm{~m}$, d. i. das anderthalbfache der für danubische Wutach und Wutach-Donau abgeleiteten Größe. Indessen erfährt dieser hohe Betrag eine angemessene Korrektur nach unten um rund $50 \mathrm{~m}$ : Unter dem Schellenberg-Plateau befinden sich heute noch annähernd $100 \mathrm{~m}$ Mittleren Muschelkalkes, eine Tatsache, die der Referent durch Hydratations-Aufschwellung (mit der Erhaltung von Steinsalz ist nicht zu rechnen) zu erklären versucht (PAuL 1958).

Solch geringe pleistozäne Erosionsleistung im danubischen Gebiet ist eine Folge der ungünstigen Vorflutverhältnisse. Aber auch hier zeigt sich - wenn man die jeweils zur Verfügung stehenden Zeiträume in Betracht zieht - die um eine volle Größenordnung höhere, während des Pleistozäns wohl die längste Zeit tätige Wirksamkeit der kaltzeitlichen Abtragungsfaktoren.

\section{Schriftenverzeichnis}

Brunnacker, K.: Über Ablauf und Altersstellung altquartärer Verschüttungen im Maintal und nächst dem Donautal bei Regensburg. - Eiszeitalter u. Gegenwart, 15, 72-80, Öhringen 1964.

Bubnoff, S. v.: Die Geschichte der Wasserscheide zwischen Wutach und Schwarza. - Ber. naturf. Ges. Freiburg, 20, 1-38, Freiburg 1913.

BüDEL, J.: Die Gliederung der Würmkaltzeit. - Würzburger Geogr. Arbeiten (Mitt. d. Geogr. Ges. Würzburg), 8, 5-45, Würzburg 1960.

Hoinkes, H.: Wir leben in einer Eiszeit. - Umschau in Wissenschaft u. Technik, 68, 26, 810-815, Frankfurt 1968.

PaUl, W.: Das Donaueschinger Ried und seine Bedeutung für die Gestaltungsgeschichte Südwestdeutschlands. - Mitteilungsbl. bad. geol. Landesanstalt 1949, 59-65, Freiburg 1950.

- : Zur Morphogenese des Schwarzwaldes. - Jh. Geol. Landesamt Baden-Württemberg, 1, 395427, Freiburg 1955.

- : Zur Morphogenese des Schwarzwaldes (II) - Jh. Geol. Landesamt Baden-Württemberg, 3, 263-359, Freiburg 1958.

- : Zur Frage der Rißvereisung der Ost- und Südostabdachung des Schwarzwaldes. - Jh. Geol. Landesamt Baden-Württemberg, 7, 423-440, Freiburg 1965.

- : Zur Frage der Rißvereisung der Ost- und Südostabdachung des Schwarzwaldes (II). - Mitt. bad. Landesv. Naturk. u. Naturschutz, N. F. 9, 309-324, Freiburg 1966.

- : In: Die Wutachschlucht (Geologie, Landschafts- und Flußgeschichte). - Monographie des bad. Landesv. Naturk. u. Naturschutz. Im Druck.

Pfannenstiel, M., \& Rahm, G.: Die Vergletscherung des Wutachtales während der Rißeiszeit. Ber. naturf. Ges. Freiburg, 53, 5-61, Freiburg 1963.

- : Nochmals zur Vergletscherung des Wutachtales während der Rißeiszeit. - Jh. Geol. Landesamt Baden-Württemberg, 8, 63-85, Freiburg 1966.

Schalch, F.: Geol. Spezialkarte d. Großherzogtums Baden nebst Erläuterungen. Blatt Bonndorf (Nr. 132), Heidelberg 1906.

- : - - Blatt Blumberg (Nr. 133), Heidelberg 1908.

WAGNer, G.: Junge Krustenbewegungen im Landschaftsbild Südwestdeutschlands. - Erdgesch. u. landesk. Abh. aus Schwaben u. Franken, 10, 300 S., Öhringen 1929.

Weprer, E.: Zur Gliederung des Glazials im Wutachgebiet. - Jber. u. Mitt. oberrh. geol. Ver. N. F. 13, 190-195, Stuttgart 1924.

Manuskr. eingeg. 3. 2. 1970.

Anschrift des Verf.: Willi Paul, 7741 Vöhrenbach, Hagenreutestraße 6. 